\title{
Half-Metallic Magnetism
}

\author{
R.A. de Groot \\ Research Institute for Materials, Nijmegen, The Netherlands \\ Materials Science Centre, Groningen, The Netherlands
}

Band structure calculations define opportunities for synthesizing novel types of an intriguing and potentially useful class of materials that is conducting for one electron spin direction and semiconducting for the other.
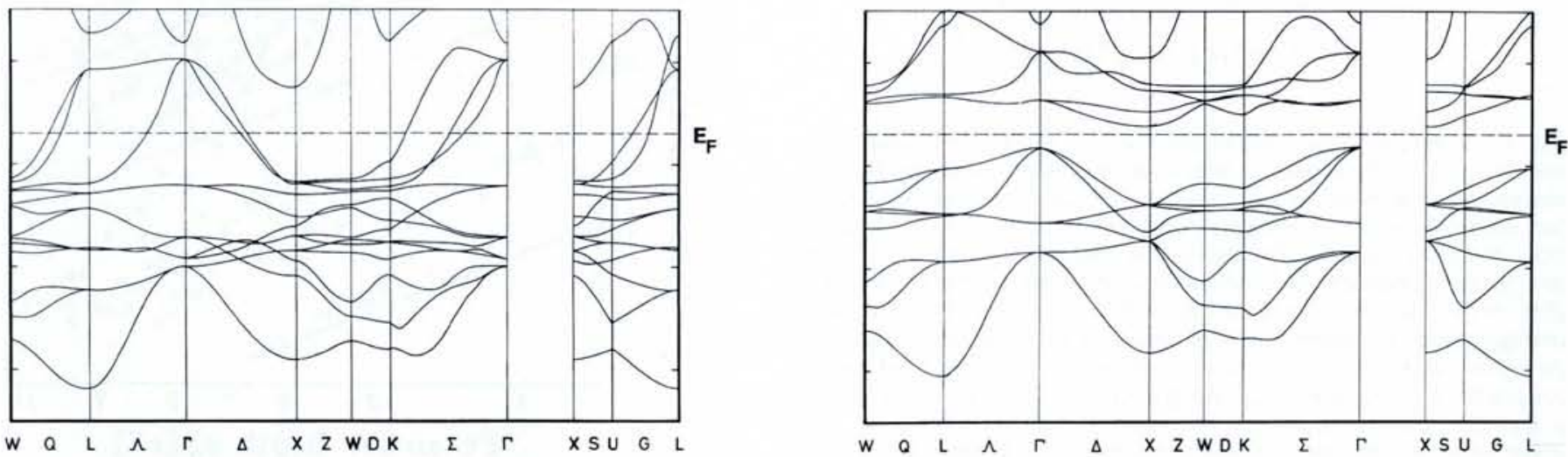

Fig. 1 - The calculated band structures of the NiMnSb half-metallic magnet for the majority (left) and minority (right) spin directions. The electron energy is plotted as function of momentum and the horizontal coordinate indicates standard points and directions in the Brillouin zone. The structure for the majority direction shows an intersection of three bands with the Fermi level typical of a metallic alloy: the structure for the minority direction displays a band gap similar to that found in semiconductors.

Two important properties of solids which have interested mankind for many years are magnetism and electrical conductivity. They manifest themselves independently so both magnetic (e.g., $\mathrm{Fe})$ and nonmagnetic (e.g., $\mathrm{Al}, \mathrm{Cu})$ metals exist, as well as magnetic (solid oxygen) and nonmagnetic (e.g., diamond) insulators. Both phenomena find their origins in the electronic structure of materials. The unique property of solids is that this structure can be characterised by the occurrence of bands of energy of allowed electron states, separated by regions which are forbidden (energy gaps). If the highest occupied level falls within a continuum, metallic conduction arises because an excitation energy is not required to move an electron to a neighbouring site: otherwise semiconducting or insulating behaviour is found. If many different states are available at the highest occupied state (the Fermi level, $E_{\mathrm{F}}$ ), i.e., a high degree of degeneracy, it may be energetically more favourable to lower the degeneracy by adopting an electronic structure which is asymmetric for the two directions of the electron spin. The number of occupied states for the two spin direc-

Professor Robert de Groot has held the Chair of Chemical Physics at the Materials Science Centre, Nijenborg 16, University of Groningen, NL-9747 AG Groningen, since 1991 and has been with the Research Institute for Materials, University of Nijmegen, since 1978. He studied solid-state chemistry at Groningen where he received his Ph.D. in 1976 before spending a year as a post-doc at IBM, Yorktown Heights, USA. tions will then be unequal and ordering of the magnetic moments thus established produces a spontaneous magnetic moment in the solid.

Asymmetry in electronic structure with respect to electron spin may lead to a situation where the Fermi level is situated in an allowed energy region for one spin direction and in an energy gap for the opposite spin direction. This represents a fairly unusual situation as metallic conduction takes place exclusively via charge carriers of one spin direction; for the opposite spin direction the material is an insulator. The phenomenon is called half-metallic magnetism.

\section{Occurrence}

The conditions required for half-metallic magnetism frequently arise if the width of the allowed energy regions (the bandwidth), controlled by the overlap of wave functions on neighbouring atoms, is smaller than the interactions which split the energy bands (e.g., the spin-orbit interaction, the electronelectron interaction and crystal fields). However, the validity of the band model is doubtful in this limit so it is unclear whether metallic conduction occurs at all. The remarkable fact is that there exist compounds with wide energy bands for one spin direction but which at the same time have a bona fide energy gap for the opposite spin direction: these are the Heussler alloys with the $C_{1}$ b structure.

The calculated dispersion curves for the energy states (band structure) of the Heussler $\mathrm{C1}_{\mathrm{b}}$ compound $\mathrm{NiMnSb}$ are shown in Fig. 1 in order to illustrate the phenomenon of half-magnetic magnetism. The elec- tronic structure of the majority spin direction is the band structure of a typical metallic alloy with three bands intersecting the Fermi energy. The derivative of the dispersion curves at the Fermi energy - a measure of the effective mass of the charge carriers is about unity, implying free-electron behaviour for this spin direction. The electronic structure of the minority spin direction is that of a semiconductor, with a band gap of about $1 \mathrm{eV}$, as in silicon.

In order to explain why this peculiar feature arises we have to consider the crystal structure of NiMnSb and the electronic configuration of its constituents. The Heussler $\mathrm{C}_{\mathrm{b}}$ structure is face-centred cubic with $\mathrm{Ni}$ occupying the $0,0,0$ positions, $M n$ the $1 / 4,1 / 4,1 / 4$ positions and $\mathrm{Sb}$ the $3 / 4,3 / 4,3 / 4$ positions. It is identical to the zinc-blende structure of the well-known III-V semiconductors, but with one of the empty sides occupied by the third constituent. Regarding the electronic configuration: for the minority spin direction, there are filled $\mathrm{Ni}$ d-shell $(5$ electrons per spin), $1 \mathrm{~s}$ and $1 \frac{1}{2} \mathrm{p}$ electrons on $\mathrm{Sb}$ and $1 \mathrm{~s}$ and $1 / 2 \mathrm{t} 2{ }_{9} \mathrm{~d}$ electrons on $\mathrm{Mn}$. This resembles the situation for a normal III-V semiconductor such as GaAs and an analysis indeed shows a very similar bonding: tetrahedral bonding in the III-V case is replaced by three-centred tetrahedral bonds in the Heussler $\mathrm{Cl}_{b}$ case.

The majority spin-electron configuration is different in two respects between the Heussler compound and a III-V semiconductor. The number of $\mathrm{d}$ electrons on $\mathrm{Mn}$ is $4 \frac{1}{2}$ and their energy is much lower in one spin direction than in the other because of exchange splitting. We find as a result a 
broad hybridised band typical of metallic alloys for the spin direction with the lower energy. The crucial ingredient is therefore the crystal structure as it allows three-centred tetrahedral bonds for the semiconducting spin direction combined with an electron configuration iso-electronic with that of III-V semiconductors. This is best exemplified by considering the other known Heussler structure, namely the $\mathrm{L}_{2}{ }_{1}$ structure. This structure is comparable to the $\mathrm{C}_{\mathrm{b}}$ structure but with the $1 / 2,1 / 2,1 / 2$ position also occupied by a $\mathrm{Ni}$ atom. Tetrahedrally coordinated atoms therefore do not exist so three-centred tetrahedral bonds cannot be formed and half-metallic properties are not found.

\section{Comparison with Experiments}

A first, indirect test of how the calculated properties compare with experiment is the magnitude of the magnetic moment. Since the number of electrons for the semiconducting spin direction is integral, as is the total number of electrons, the magnetic moment of a half-metallic system is necessarily also integral. For NiMnSb, this moment is calculated to be four and neutron diffraction experiments give the value of 4.0 .

Another indirect confirmation of the validity of the calculations is given by analysing the electrical conductivity. One usually finds in magnetic systems at low temperatures a term proportional to the temperature $T$ squared. This term originates from so-called onemagnon scattering where both a localised magnetic moment and a conduction electron reverse their spin directions simultaneously. Such a scattering mechanism is impossible when there are only states of one spin direction for conduction electrons. Indeed, it has been found for NiMnSb that the $T^{2}$ term in the low-temperature resistivity is absent.

The most direct confirmation of the model comes from positron annihilation experiments. Two $\gamma$ quanta are produced when an electron and its anti-particle, the positron, annihilate. As momentum is conserved in the process, deviations from exactly opposing directions for the emerging $\gamma$ quanta are a direct measure of the momenta of the annihilated particles. A positron loses its momentum almost instantaneously in a solid so an analysis of the direction of the $\gamma$ quanta in positron annihilation measures directly the momentum of an electron in the material. For a metal, one finds an abrupt cut-off of the $\gamma$-rays for angles corresponding to the momentum of an energy band crossing the Fermi energy because states beyond this limit are unoccupied. The cut-off therefore leads directly to a measurement of the momenta of the electron states with the Fermi energy, i.e., the Fermi surface. Positron annihilation is spin-selective because the positron source is spin polarized due to the non-parity conservation of $\gamma$-decay and the fact that the annihilation process depends on the magnetic orientation of the two annihilating particles: an electron-positron pair in a triplet state (electron and positron spin parallel) leads to the production of three $\gamma$ quanta instead of two. It was proven unambiguously this way that NiMnSb has a Fermi surface for only one spin direction, i.e., it is metallic for only one spin direction.
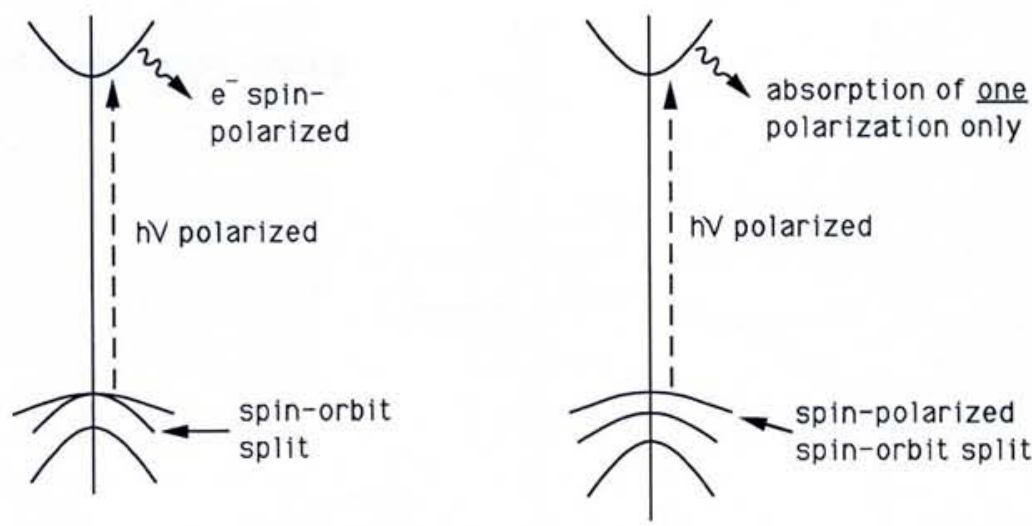

Fig. 2 - The Magneto-Optic Kerr Effect (MOKE) in the PtMnSb half-metallic system is the complementary process to the production of spin-polarized electrons by exciting electrons in GaAs under polarized light. Left: excitation with polarized light of electrons from the top of a valence band with spin-orbit splitting produces spin-polarized electrons.

Right: excitation from a spin-polarized, spin-orbit split top of the valence band in the half-metallic magnet leads to the absorption of one polarization of light only.

\section{Magneto-Optic Properties}

Research leading to the discovery of halfmetallic magnetism was in fact triggered by the unusual magneto-optic properties of $\mathrm{PtMnSb}$. If linearly polarized light is reflected by a surface of a magnetic material, the reflected light is found to be elliptically polarized with the rotation of the polarization angle and the degree of ellipticity determined by the orientation of the magnetization of the material. This phenomenon is known as the Magneto-Optic Kerr Effect (MOKE).

The material with the largest known roomtemperature MOKE is PtMnSb which is isoelectronic and iso-structural with $\mathrm{NiMnSb}$, and is also a half-metallic ferromagnet. The unusual MOKE properties follow directly from its half-metallic properties: at the band gap of the semiconducting spin direction, only transitions for one (circular) polarization direction are possible. Since linearly polarized light can be decomposed into equal contributions of right and left circularly polarized light, a large MOKE results (Fig. 2). It should be stressed that half-metallic properties by themselves are no guarantee of a high MOKE: the electronic transition across the gap in PtMnSb is quite strong, unlike in NiMnSb which only shows a modest MOKE.

\section{Spin-Polarized Electron Probes}

A possible application of half-metallic magnets is as a source of spin-polarized electrons in tunnelling experiments such as the scanning tunnelling microscope (STM). The STM measures not only the structure of a surface but also the information obtained by measuring the tunnelling current versus the applied voltage. So it also gives, in principle, information about the density of states at the surface. Using a half-metallic tip as a STM probe should enable one to determine the density of states as a function of spin direction. This is important for magnetic surfaces, absorbed magnetic atoms, etc.

Spin-resolved tunnelling was realised recently using a chromium oxide tip on a stepped $\mathrm{Cr}$ surface. The surface of this antiferromagnetic material shows steps with opposite spin directions. The application of a half-magnetic tip to study the surfaces of permanent magnets leads to complications.

\section{Other Systems}

As has already been mentioned, in the case of very narrow bands the phenomena of half-metallic magnetism should occur quite frequently, so we expect it to be widespread in rare-earth and actinide compounds. Many of these materials do indeed show very interesting magneto-optical properties. However, as far as conduction is concerned, it is doubtful that conduction is possible here and that the unusual transport properties of half-metallic magnets occur.

\section{Oxides}

A borderline case is found in some $3 \mathrm{~d}$ transition-metal oxides. $\mathrm{Fe}_{3} \mathrm{O}_{4}$ above $120 \mathrm{~K}$ is, according to band structure calculations, a half-metallic ferrimagnet with rather narrow bands, but which conducts electricity. The case of $\mathrm{CrO}_{2}$ is more complicated. Band structure calculations show it to be half-metallic with rather narrow bands, while photo-emission and inverse photo-emission measurements lead to the conclusion that it is a semiconductor with a $2 \mathrm{eV}$ band gap. $\mathrm{CrO}_{2}$ also conducts electricity, but a very dirty semiconductor also does this. Initial STM measurements indicated evidence for gaps in both directions thus favouring conclusions derived from photo-emission. Clearly more research is needed on this compound - both from the point-of-view of calculation, to determine the electronic structure of the surface and the influence of localised excitation processes in photoemission; and from the experimental pointof-view, to carefully establish the oxygen content of the surface under the ultra-high vacuum conditions present during photoemission experiments.

\section{TMMnSb series}

Regarding Heussler alloys other than PtMnSb and NiMnSb, the transition-metal (TM) series CoMnSb, FeMnSb, MnMnSb and $\mathrm{CrMnSb}$ have been investigated. The calculated band structures are most interesting. Starting from NiMnSb, every step in the series is characterised by a lowering of the number of valence electrons by one. In a rigid-band scheme (lowering $E_{\mathrm{F}}$ but keeping the band structure unaffected), the half- 


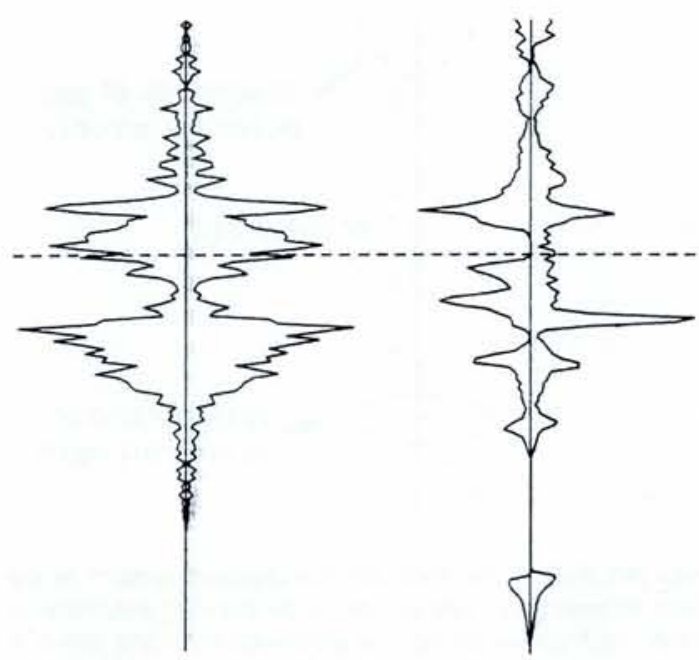

Fig. 3 - The calculated density of states (energy as a function of state density) plotted back-to-back for the two electron spin directions in the normal antiferromagnet $\mathrm{Cr}$ (left) and in the half-metallic antiferromagnet $\mathrm{CrMnSb}$ (right). Note the complete symmetry in the case of $\mathrm{Cr}$ and the $0.8 \mathrm{eV}$ energy gap for one spin direction at the Fermi energy (dotted line) in the case of $\mathrm{CrMnSb}$. metallic properties are expected to have disappeared on reaching CoMnSb. This is not what happens. The semiconducting spin direction is hardly affected across the series so all of these Heussler compounds are half-metallic. This results in a total magnetic moment of 3 for $\mathrm{CoMnSb}$ while FeMnSb has a net moment of 2 . The stability of the latter compound with its very small moment results from the build up across the series of a magnetic moment antiparallel with respect to the $\mathrm{Mn}$ moment on the transition metal other than Mn. So FeMnSb has a moment of 2 , reducing to 1 for $M n M n S b$ and 0 for $\mathrm{CrMnSb}$.

This last compound is exceptionally exotic: although a bona fide half-metallic system (Fig. 3), it does not have a magnetization! This is because the moment on Mn is cancelled by that on $\mathrm{Cr}$, i.e., it is antiferromaentirely different nature to that of conventional antiferromagnets, where symmetry relates sites with opposite spin so half-metallic properties cannot arise as identical densities of states exist for the two spin directions. For $\mathrm{CrMnSb}$, the exact cancellation of the local magnetic moments does not originate from a symmetry relation but from its half-metallic properties: the total moment has to be integral. Clearly, such a material would be ideal as a tip material in tunnelling experiments since it does not perturb the

Unfortunately, Nature is not so kind as to yield all of this series of materials in the Heussler structure. CoMnSb forms a superstructure with quite different magnetic moments; FeMnSb cannot be prepared in the Heussler structure with the required stoichiometry; MnMnSb and $\mathrm{CrMnSb}$ in fact exist, but in very different crystal structures that do not obey the requirements for wideband half-metallic behaviour (indeed, they are not even half-metallic).

\section{Multilayers}

The possibility of preparing materials under conditions well-removed from thermodynamic equilibrium represents a formidable challenge in solid-state physics research. The enormous richness realised in organic chemistry and biology using very few elements originates from the fact that virtually all of their compounds are formed at modegnetic. But its antiferromagnetism is of an substrate magnetically. rate temperatures. Molecular beam epitaxy (MBE) is one method which allows the synthesis of solids where man rather than equilibrium thermodynamics decides where atoms go. As the degree of control is presently limited to one dimension, we shall consider multilayer systems.

The two relevant questions are whether one can predict multilayer systems which exhibit interesting magneto-optical properties and/or half-metallic antiferromagnetism. If a multilayer system is made up of layers of a metal and a semiconductor it is clear that the metallic properties will convert into semiconducting properties somewhere near the layer interfaces, at a position determined by the electronic structure of both the metal and semiconductor. For a strongly magnetic metal, the electronic structure is substantially different for the two spin directions. Hence, it can be expected that the position where the band gap moves upwards will be spin-dependent. It is furthermore plausible that the multilayer system will have a volume where half-metallic properties exist. What remains to be determined is how large a fraction of the volume of the system is half-metallic.

The calculated electronic structure of a multilayer system composed of monolayers that the entire multilayer structure is halfmetallic as there is an intersection with $E_{\mathrm{F}}$ for one spin direction whereas the other spin direction shows a gap at $E_{\mathrm{F}}$. Nevertheless, the total magnetic moment is zero.

In considering the prospects for realising a half-metallic antiferromagnetic multilayer, one must recognise that the Heussler $\mathrm{C}_{1}$ b structure itself is a naturally occurring multilayer system. In the case of CrMnSb, hexagonal layers of $\mathrm{Sb}, \mathrm{Cr}, \mathrm{Mn}$ followed by vacancies followed by $\mathrm{Sb}$, etc. are found along the $<111>$ direction. A practical complication may be the need to incorporate these vacancy layers during MBE processing, although it cannot be excluded that $\mathrm{CrMnSb}$ in the Heussler structure is sufficiently (meta)stable for the vacancy layer to form by itself. Alternatively, the $<001>$ direction in $\mathrm{CrMnSb}$ may be considered as a multilayer system composed of layers of $\mathrm{Cr}$, $\mathrm{MnSb}, \mathrm{Cr}$, etc. so this sequence should also be examined in MBE experiments.

Another alternative exists in the event that the vacancy structure does not form. The vacancy is not essential as long as the site is occupied by an atom that does not perturb the three-centred tetrahedral bonds for the semiconducting spin direction. Thus, if we replace pentavalent $\mathrm{Sb}$ by tetravalent $\mathrm{Sn}$ and fill the $1 / 2,1 / 2,1 / 2$ position with a monovalent metal, half-metallic antiferromagnetism may still survive. This point is illustrated by the calculated band structure of $\mathrm{CrMn}$ $\mathrm{NaSn}$, consisting of hexagonal layers of the constituents in the order given by the formula. Fig. 4 demonstrates that this system is indeed a half-metallic antiferromagnet. It should however be pointed out that none of these hypothetical, but intriguing and potentially useful, multilayer systems have yet been realised experimentally.

\section{FURTHER READING}

Wiesendanger R., et al., Phys. Rev. Lett. 65 (1990) 247

Wiesendanger R., et al., Science 255 (1992) 583. of the semiconductor $\mathrm{PbO}$ and of $\mathrm{Fe}$ shows

De Groot R.A., Physica B 172 (1991) 45.
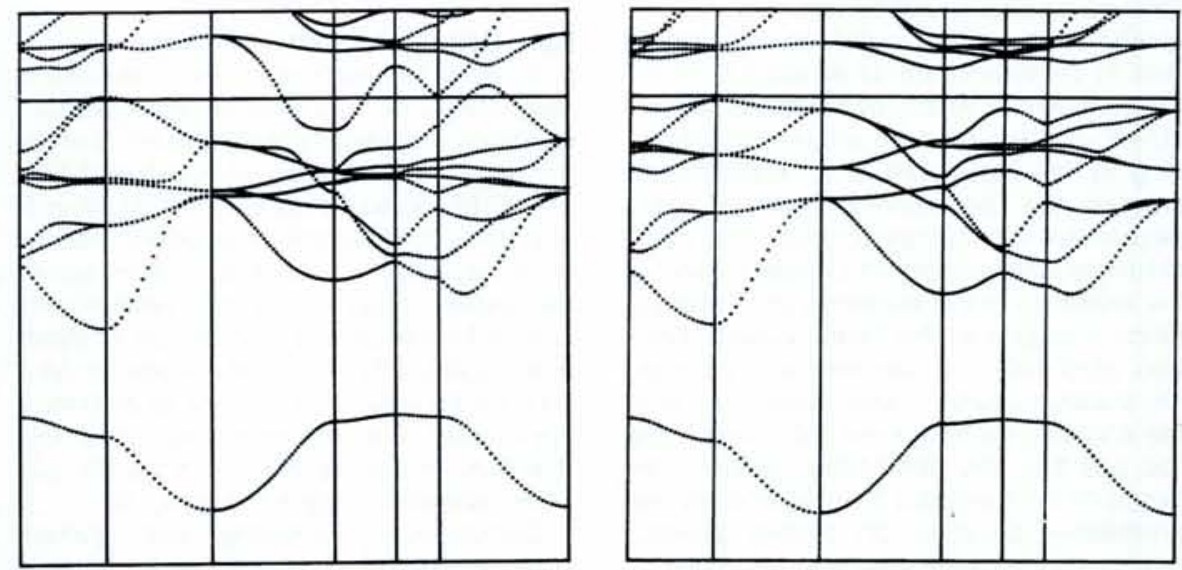

Fig. 4-Calculated band structures (same coordinates as Fig. 1) for the two spin directions in an infinite multilayer system composed of alternating $\mathrm{Cr}-\mathrm{Mn}-\mathrm{Na}$-Sn monolayers with hexagonal structures. The band structure for one spin direction (on the left) shows a metallic intersection of the Fermi energy while the other shows a gap at $E_{F}$. The material is nonetheless antiferromagnetic, the total magnetic moment being zero, so it would be ideal for a STM probe. 